\title{
Boundedness and compactness of a class of Hardy type operators
}

\author{
Akbota M Abylayeva', Ryskul Oinarov ${ }^{1}$ and Lars-Erik Persson ${ }^{2,3 *}$
}

"Correspondence: larserik@ltu.se
2Department of Engineering
Sciences and Mathematics, Luleå
University of Technology, Luleå,
97187 , Sweden
${ }^{3}$ UiT, The Artic University of Norway,
Tromsø, Norway
Full list of author information is
available at the end of the article

*Correspondence: larserik@ltu.se ${ }^{2}$ Department of Engineering Sciences and Mathematics, Lulea 97187, Sweden

佂, The Artic University of Norway, Full list of author information is alle at the end of the article

\begin{abstract}
We establish characterizations of both boundedness and of compactness of a general class of fractional integral operators involving the Riemann-Liouville, Hadamard, and Erdelyi-Kober operators. In particular, these results imply new results in the theory of Hardy type inequalities. As applications both new and well-known results are pointed out.

MSC: $26 \mathrm{~A} 33 ; 26 \mathrm{D} 10 ; 47 \mathrm{G} 10$

Keywords: inequalities; Hardy type inequalities; fractional integral operator; Riemann-Liouville operator; Hadamard operator; Erdelyi-Kober operator; boundedness; compactness
\end{abstract}

\section{Introduction}

Let $I=(a, b), 0 \leq a<b \leq \infty$. Let $v$ and $u$ be almost everywhere positive functions, which are locally integrable on the interval $I$.

Let $0<p<\infty$ and $\frac{1}{p}+\frac{1}{p^{\prime}}=1$. Denote by $L_{p, v} \equiv L_{p}(v, I)$ the set of all functions $f$ measurable on $I$ such that $\|f\|_{p, v}:=\left(\int_{a}^{b}|f(x)|^{p} v(x) d x\right)^{\frac{1}{p}}<\infty$.

Let $W$ be a non-negative, strictly increasing and locally absolutely continuous function on $I$. Suppose that $\frac{d W(x)}{d x}=w(x)$, a.e. $x \in I$.

We consider the Hardy type operator $T_{\alpha, \beta}$ defined by

$$
T_{\alpha, \beta} f(x):=\int_{a}^{x} \frac{u(s) W^{\beta}(s) f(s) w(s) d s}{(W(x)-W(s))^{1-\alpha}}, \quad x \in I
$$

When $u \equiv 1$ and $\beta=0$ the operator $T_{\alpha, \beta}$ is called the fractional integral operator of a function $f$ with respect to a function $W([1], \mathrm{p} .248)$. When $u \equiv 1$ and $W(x)=x$ the operator (1.1) becomes the Riemann-Liouville operator $I_{\alpha}$ defined by

$$
I_{\alpha} f(x):=\int_{a}^{x} \frac{s^{\beta} f(s) d s}{(x-s)^{1-\alpha}}
$$

When $u \equiv 1$ and $W(x) \equiv \ln \frac{x}{a}, a>0$, this operator is the Hadamard operator $\mathcal{H}_{\alpha}$ defined by

$$
\mathcal{H}_{\alpha} f(x):=\int_{a}^{x} \frac{\left(\ln \frac{s}{a}\right)^{\beta} f(s) d s}{s\left(\ln \frac{x}{s}\right)^{1-\alpha}} .
$$

(c) The Author(s) 2016. This article is distributed under the terms of the Creative Commons Attribution 4.0 International License (http://creativecommons.org/licenses/by/4.0/), which permits unrestricted use, distribution, and reproduction in any medium, provided you give appropriate credit to the original author(s) and the source, provide a link to the Creative Commons license, and indicate if changes were made. 
Moreover, when $u \equiv 1$ and $W(x)=x^{\sigma}, \sigma>0$, we get the operator $E_{\alpha, \beta}$ of Erdelyi-Kober type ([1], p.246) defined by

$$
E_{\alpha, \beta} f(x):=\sigma \int_{a}^{x} \frac{f(s) s^{\sigma \beta+\sigma-1} d s}{\left(x^{\sigma}-s^{\sigma}\right)^{1-\alpha}}
$$

There are a lot of works devoted to the mapping properties of the Riemann-Liouville operator $I_{\alpha}$. Two-weighted estimates of the operator $I_{\alpha}$ of the order $\alpha>1$ in weighted Lebesgue spaces were first obtained in the papers [2] and [3]. The singular case $0<\alpha<1$ was studied with different restrictions in [4-9] and some others. The most general results among them are given in [5] and [9] under the assumption that one of the weight functions is increasing or decreasing.

In this work we investigate the problems of boundedness and compactness of the operator $T_{\alpha, \beta}$ defined by (1.1) from $L_{p, w}$ to $L_{q, v}$ when $0<\alpha<1$. When $\alpha>1$ the results follow from the results in [10].

The operator $T_{\alpha, \beta}$ was studied in [11] and [12] when $u \equiv 1, \beta=0$ and $u \equiv 1, \beta>-\frac{1}{p^{\prime}}$, respectively.

Due to the non-negativity and monotone increase of the function $W$ the limit $\lim _{x \rightarrow a^{+}} W(x) \equiv W(a) \geq 0$ exists.

We also consider the Hardy type operator $T_{\alpha, \beta}^{0}$ defined by

$$
T_{\alpha, \beta}^{0} f(x):=\int_{a}^{x} \frac{u(s) W_{0}^{\beta}(s) f(s) w(s) d s}{\left(W_{0}(x)-W_{0}(s)\right)^{1-\alpha}}, \quad x \in I
$$

where $W_{0}(x)=W(x)-W(a)$.

Since we also suppose that $\beta \geq 0$, for $f \geq 0$ we have $T_{\alpha, \beta} f(x) \approx T_{\alpha, \beta}^{0} f(x)+W(a) T_{\alpha, 0}^{0} f(x)$, where the equivalence constants do not depend on $x$ and $f$. Therefore, without loss of generality, we can assume that $W(a)=0$. For short writing we denote by $\|K\|$ the norm of a linear operator $K$ acting from one normalized space to another, since from the context we shall in each case clearly see which spaces the operator is acting between.

The paper is organized as follows: In order not to disturb our discussions later on some auxiliary statements are given in Section 2 . The main results concerning the boundedness of operator $T_{\alpha, \beta}$, including the corresponding Hardy type inequalities, can be found in Section 3. The main results about the compactness are presented in Section 4. Moreover, in Section 5 some similar results for the dual operator $T_{\alpha, \beta}^{0}$ are given. Finally, Section 6 is reserved for some applications (both new and well-known results).

Conventions The indeterminate form $0 \cdot \infty$ is assumed to be zero. The relations $A \ll B$ and $A \gg B$, respectively, mean $A \leq c B$ and $A \geq c B$, where a positive constant $c$ can be dependent only on the parameters $p, q, \alpha$ and $\beta$. The relation $A \approx B$ is interpreted as $A \ll B \ll A$. The set of all integers is denoted by $Z$. Moreover, $\chi_{(c, a)}(\cdot)$ is the characteristic function of the interval $(c, a) \subset I$.

\section{Auxiliary statements}

To prove the main results we shall need some auxiliary results from the standard literature on Hardy type inequalities (see [13] and [14]). 
Together with the operator (1.1) we consider the Hardy type operator $H_{\alpha, \beta}$ defined by

$$
H_{\alpha, \beta} f(x)=\frac{1}{W^{1-\alpha}(x)} \int_{a}^{x} u(s) W^{\beta}(s) f(s) w(s) d s
$$

It is easy to see that for $f \geq 0$ we have

$$
T_{\alpha, \beta} f(x) \geq H_{\alpha, \beta} f(x), \quad \forall x \in I .
$$

The problem of boundedness of operators of the form (2.1) in weighted Lebesgue spaces have been very well studied. The history and development of Hardy type inequalities with relevant references can be found in [13].

In view of [15] the following statements are consequences of Theorem 5 of [13].

Lemma 2.1 Let $1<p \leq q<\infty$ and let the operator $H_{\alpha, \beta}$ be defined by (2.1). Then the inequality

$$
\left(\int_{a}^{b}\left(H_{\alpha, \beta} f(x)\right)^{q} v(x) d x\right)^{\frac{1}{q}} \leq C\left(\int_{a}^{b}(f(x))^{p} w(x) d x\right)^{\frac{1}{p}}
$$

holds if and only if

$$
A_{\alpha, \beta}=\sup _{z \in I}\left(\int_{a}^{z} u^{p^{\prime}}(s) W^{p^{\prime} \beta}(s) w(s) d s\right)^{\frac{1}{p^{\prime}}}\left(\int_{z}^{b} W^{q(\alpha-1)}(x) v(x) d x\right)^{\frac{1}{q}}<\infty .
$$

Moreover, $C \approx A_{\alpha, \beta}$.

Lemma 2.2 Let $0<q<p<\infty, p>1$ and let the operator $H_{\alpha, \beta}$ be defined by (2.1). Then the inequality (2.3) holds if and only if

$$
\begin{aligned}
B_{\alpha, \beta}= & \left(\int_{a}^{b}\left(\int_{z}^{b} W^{q(\alpha-1)}(x) v(x) d x\right)^{\frac{p}{p-q}}\right. \\
& \left.\times\left(\int_{a}^{z} u^{p^{\prime}}(s) W^{p^{\prime} \beta}(s) w(s) d s\right)^{\frac{p(q-1)}{p-q}} u^{p^{\prime}}(z) W^{p^{\prime} \beta}(z) w(z) d z\right)^{\frac{p-q}{p q}}<\infty .
\end{aligned}
$$

Moreover, $C \approx B_{\alpha, \beta}$.

Remark 2.3 In the case $1<q<p<\infty, p>1$ it is well known and easy to prove that the value $B_{\alpha, \beta}$ is equivalent to the value

$$
\begin{aligned}
\widetilde{B}_{\alpha, \beta}= & \left(\int_{a}^{b}\left(\int_{z}^{b} W^{q(\alpha-1)}(x) v(x) d x\right)^{\frac{q}{p-q}}\right. \\
& \left.\times\left(\int_{a}^{z} u^{p^{\prime}}(s) W^{p^{\prime} \beta}(s) w(s) d s\right)^{\frac{q(p-1)}{p-q}} W^{q(\alpha-1)}(z) v(z) d z\right)^{\frac{p-q}{p q}} .
\end{aligned}
$$




\section{Boundedness of the operator $T_{\alpha, \beta}$}

The main results in this section read as follows.

Theorem 3.1 Let $0<\alpha<1,1<p \leq q<\infty$ and $\beta \geq 0$. Let $u$ be a non-increasing function on I. Then the operator $T_{\alpha, \beta}$ defined by (1.1) is bounded from $L_{p, w}$ to $L_{q, v}$ if and only if $A_{\alpha, \beta}<\infty$. Moreover, $\left\|T_{\alpha, \beta}\right\| \approx A_{\alpha, \beta}$.

Theorem 3.2 Let $0<\alpha<1,0<q<p<\infty, p>\frac{1}{\alpha}$ and $\beta \geq 0$. Let $u$ be a non-increasing function on $I$. Then the operator $T_{\alpha, \beta}$ is bounded from $L_{p, w}$ to $L_{q, v}$ if and only if $B_{\alpha, \beta}<\infty$. Moreover, $\left\|T_{\alpha, \beta}\right\| \approx B_{\alpha, \beta}$.

These two theorems can be reformulated as the following new information in the theory of Hardy type inequalities.

Theorem 3.3 Let $0<\alpha<1, \beta \geq 0$ and $u$ be a non-increasing function on I. Then the inequality

$$
\left(\int_{a}^{b}\left(T_{\alpha, \beta} f(x)\right)^{q} v(x) d x\right)^{\frac{1}{q}} \leq C\left(\int_{a}^{b}(f(x))^{p} w(x) d x\right)^{\frac{1}{p}}
$$

holds if and only if

(a) $A_{\alpha, \beta}<\infty$ for the case $1<p \leq q<\infty$,

(b) $B_{\alpha, \beta}<\infty$ for the case $0<q<p<\infty, p>\frac{1}{\alpha}$.

Moreover, for the best constant $C$ in (3.1) it yields $C \approx A_{\alpha, \beta}$ in case (a) and $C \approx B_{\alpha, \beta}$ in case (b).

Proof of Theorem 3.1 Necessity. Let the operator $T_{\alpha, \beta}$ be bounded from $L_{p, w}$ to $L_{q, v}$. Then, in view of (2.2), the operator $H_{\alpha, \beta}$ is bounded from $L_{p, w}$ to $L_{q, v}$, and $\left\|T_{\alpha, \beta}\right\| \geq\left\|H_{\alpha, \beta}\right\|$. Consequently, by Lemma 2.1 we have $A_{\alpha, \beta}<\infty$ and

$$
\left\|T_{\alpha, \beta}\right\| \gg A_{\alpha, \beta}
$$

Sufficiency. Since the function $W$ is continuous and strictly increasing on $I$ and $W(a)=$ 0 , for any $k \in Z$ we can define $x_{k}:=\sup \left\{x: W(x) \leq 2^{k}, x \in I\right\}$. We obtain a sequence of points $\left\{x_{k}\right\}_{k>-\infty}$ such that $0<x_{k} \leq x_{k+1}, \forall k \in Z$, and if $x_{k}<b$, then $W\left(x_{k}\right)=2^{k}, 2^{k} \leq W(x) \leq$ $2^{k+1}$ for $x_{k} \leq x \leq x_{k+1}, \int_{x_{k-1}}^{x_{k}} w(s) d s=2^{k-1}$, and if $x_{k+1}=b$, then $\int_{x_{k}}^{x_{k+1}} w(s) d s \leq 2^{k}$. These facts will be used below without reminders. We assume that $I_{k}=\left[x_{k}, x_{k+1}\right), k \in Z, Z=\{k: k \in$ $\left.Z, I_{k} \neq \emptyset\right\}$. Then $Z_{0} \subseteq Z$ and $I=\bigcup_{k \in Z} I_{k}=\bigcup_{k \in Z_{0}} I_{k}$. Since $I_{k}=\emptyset, \forall k \in Z \backslash Z_{0}$, and integrals over these intervals are equal to zero, in the sequel, without loss of generality, we can suppose that $Z=Z_{0}$.

Let $A_{\alpha, \beta}<\infty$. We need to prove that the inequality

$$
\left\|T_{\alpha, \beta} f\right\|_{q, v} \ll A_{\alpha, \beta}\|f\|_{p, w}, \quad f \in L_{p, w}
$$

holds, which means $\left\|T_{\alpha, \beta}\right\| \ll A_{\alpha, \beta}$ and, together with (3.2), this gives

$$
\left\|T_{\alpha, \beta}\right\| \approx A_{\alpha, \beta}
$$


Let $f \geq 0$. Using the relation $I=\bigcup_{k} I_{k}$, we have

$$
\begin{aligned}
\left\|T_{\alpha, \beta} f\right\|_{q, v}^{q}= & \sum_{k} \int_{x_{k}}^{x_{k+1}} v(x)\left(\int_{a}^{x} \frac{u(s) W^{\beta}(s) f(s) w(s) d s}{(W(x)-W(s))^{1-\alpha}}\right)^{q} d x \\
= & \sum_{k} \int_{x_{k}}^{x_{k+1}} v(x)\left[\left(\int_{a}^{x_{k-1}}+\int_{x_{k-1}}^{x}\right) \frac{u(s) W^{\beta}(s) f(s) w(s) d s}{(W(x)-W(s))^{1-\alpha}}\right]^{q} d x \\
\ll & \sum_{k} \int_{x_{k}}^{x_{k+1}} v(x)\left(\int_{a}^{x_{k-1}} \frac{u(s) W^{\beta}(s) f(s) w(s) d s}{(W(x)-W(s))^{1-\alpha}}\right)^{q} d x \\
& +\sum_{k} \int_{x_{k}}^{x_{k+1}} v(x)\left(\int_{x_{k-1}}^{x} \frac{u(s) W^{\beta}(s) f(s) w(s) d s}{(W(x)-W(s))^{1-\alpha}}\right)^{q} d x:=J_{1}+J_{2} .
\end{aligned}
$$

We now estimate $J_{1}$ and $J_{2}$ separately. Using the monotonicity of $W$ we find that

$$
\begin{aligned}
J_{1} & =\sum_{k} \int_{x_{k}}^{x_{k+1}} v(x)\left(\int_{a}^{x_{k-1}} \frac{u(s) W^{\beta}(s) f(s) w(s) d s}{(W(x)-W(s))^{1-\alpha}}\right)^{q} d x \\
& \leq \sum_{k} \int_{x_{k}}^{x_{k+1}} v(x)\left(\int_{a}^{x_{k-1}} \frac{u(s) W^{\beta}(s) f(s) w(s) d s}{\left(W\left(x_{k}\right)-W\left(x_{k-1}\right)\right)^{1-\alpha}}\right)^{q} d x \\
& =2^{2 q(1-\alpha)} \sum_{k} \int_{x_{k}}^{x_{k+1}} v(x)\left(\frac{1}{2^{k+1}}\right)^{q(1-\alpha)}\left(\int_{a}^{x_{k-1}} u(s) W^{\beta}(s) f(s) w(s) d s\right)^{q} d x \\
& \ll \sum_{k} \int_{x_{k}}^{x_{k+1}} v(x) W^{q(\alpha-1)}(x)\left(\int_{a}^{x} u(s) W^{\beta}(s) f(s) w(s) d s\right)^{q} d x \leq\left\|H_{\alpha, \beta} f\right\|_{q, v}^{q} .
\end{aligned}
$$

Hence, by Lemma 2.1 we get

$$
J_{1} \ll A_{\alpha, \beta}^{q}\|f\|_{p, w}^{q}
$$

Moreover, by using Hölder's inequality and the fact that the function $\mathrm{u}$ is increasing, we obtain

$$
\begin{aligned}
J_{2}= & \sum_{k} \int_{x_{k}}^{x_{k+1}} v(x)\left(\int_{x_{k-1}}^{x} \frac{u(s) W^{\beta}(s) f(s) w(s) d s}{(W(x)-W(s))^{1-\alpha}}\right)^{q} d x \\
\leq & \sum_{k} \int_{x_{k}}^{x_{k+1}} v(x)\left(\int_{x_{k-1}}^{x} f^{p}(s) w(s) d s\right)^{\frac{q}{p}}\left(\int_{x_{k-1}}^{x} \frac{u^{p^{\prime}}(s) W^{p^{\prime} \beta}(s) w(s) d s}{(W(x)-W(s))^{p^{\prime}(1-\alpha)}}\right)^{\frac{q}{p^{\prime}}} d x \\
\leq & \sum_{k}\left(\int_{x_{k-1}}^{x_{k+1}} f^{p}(s) w(s) d s\right)^{\frac{q}{p}} u^{q}\left(x_{k-1}\right) \\
& \times \int_{x_{k}}^{x_{k+1}} v(x)\left(\int_{a}^{x} \frac{W^{p^{\prime} \beta}(s) w(s) d s}{(W(x)-W(s))^{p^{\prime}(1-\alpha)}}\right)^{\frac{q}{p^{\prime}}} d x .
\end{aligned}
$$

A change of variables $W(s)=W(x) t$ in the last integral, implies that

$$
\int_{a}^{x} \frac{W^{p^{\prime} \beta}(s) w(s) d s}{(W(x)-W(s))^{p^{\prime}(1-\alpha)}}=\frac{W^{p^{\prime} \beta+1}(x)}{W^{p^{\prime}(1-\alpha)}(x)} \int_{0}^{1} t^{p^{\prime} \beta}(1-t)^{p^{\prime}(\alpha-1)} d t
$$


Since $\beta \geq 0, \alpha>\frac{1}{p}$, the Euler beta function $\int_{0}^{1} t^{p^{\beta}} \beta(1-t)^{p^{\prime}(\alpha-1)} d t$ converges. Consequently, from (3.6) and (3.7) it follows that

$$
\begin{aligned}
& J_{2} \ll \sum_{k}\left(\int_{x_{k-1}}^{x_{k+1}} f^{p}(s) w(s) d s\right)^{\frac{q}{p}} u^{q}\left(x_{k-1}\right) \int_{x_{k}}^{x_{k+1}} v(x) \frac{W^{\frac{q}{p^{\prime}}\left(p^{\prime} \beta+1\right)} d x}{W^{q(1-\alpha)}(x)} \\
& \leq \sum_{k}\left(\int_{x_{k-1}}^{x_{k+1}} f^{p}(s) w(s) d s\right)^{\frac{q}{p}} u^{q}\left(x_{k-1}\right) W^{\frac{q}{p^{\prime}}\left(p^{\prime} \beta+1\right)}\left(x_{k+1}\right) \int_{x_{k}}^{x_{k+1}} v(x) W^{q(\alpha-1)}(x) d x \\
&= 2^{2\left(q \beta+\frac{q}{p^{\prime}}\right)} \sum_{k}\left(\int_{x_{k-1}}^{x_{k+1}} f^{p}(s) w(s) d s\right)^{\frac{q}{p}} \\
& \times u^{q}\left(x_{k-1}\right) W^{\frac{q}{p^{\prime}}\left(p^{\prime} \beta+1\right)}\left(x_{k-1}\right) \int_{x_{k}}^{x_{k+1}} v(x) W^{q(\alpha-1)}(x) d x \\
& \ll \sum_{k}\left(\int_{x_{k-1}}^{x_{k+1}} f^{p}(s) w(s) d s\right)^{\frac{q}{p}} \\
& \times u^{q}\left(x_{k-1}\right)\left(\int_{a}^{x_{k-1}} W^{p^{\prime} \beta}(s) w(s) d s\right)^{\frac{q}{p^{\prime}}} \int_{x_{k}}^{x_{k+1}} v(x) W^{q(\alpha-1)}(x) d x \\
& \leq \sum_{k}\left(\int_{x_{k-1}}^{x_{k+1}} f^{p}(s) w(s) d s\right)^{\frac{q}{p}} \\
& \times\left(\int_{a}^{x_{k}} u^{p^{\prime}}(s) W^{p^{\prime} \beta}(s) w(s) d s\right)^{\frac{q}{p^{\prime}}} \int_{x_{k}}^{b} v(x) W^{q(\alpha-1)}(x) d x \\
& \leq A_{\alpha, \beta}^{q} \sum_{k}\left(\int_{x_{k-1}}^{x_{k+1}} f^{p}(s) w(s) d s\right)^{\frac{q}{p}} \leq A_{\alpha, \beta}^{q}\left(\sum_{k}^{q} \int_{x_{k-1}}^{x_{k+1}} f^{p}(s) w(s) d s \|_{p, w^{*}}^{q}\right. \\
& \frac{q}{p}
\end{aligned}
$$

By combining (3.4), (3.5) and (3.8) we obtain (3.3). The proof is complete.

Proof of Theorem 3.2 Necessity. Similarly to the proof of Theorem 3.1 and the estimate

$$
\left\|T_{\alpha, \beta}\right\| \gg B_{\alpha, \beta}
$$

it follows from (2.2) and Lemma 2.2.

Sufficiency. Let $B_{\alpha, \beta}<\infty$. If we show that $\left\|T_{\alpha, \beta}\right\| \ll B_{\alpha, \beta}$, then this fact and (3.9) imply that $\left\|T_{\alpha, \beta}\right\| \approx B_{\alpha, \beta}$. Next, we use relation (3.4). For the estimate $J_{1}$ we have obtained $J_{1} \ll$ $\left\|H_{\alpha, \beta} f\right\|_{q, v}^{q}$. Hence, by Lemma 2.2 we obtain

$$
J_{1} \ll B_{\alpha, \beta}^{q}\|f\|_{p, w}^{q}
$$

Moreover, from Theorem 3.1, obvious estimates and Hölder's inequality it follows that

$$
\begin{aligned}
J_{2} \ll & \sum_{k}\left(\int_{x_{k-1}}^{x_{k+1}} f^{p}(s) w(s) d s\right)^{\frac{q}{p}} \\
& \times u^{q}\left(x_{k-1}\right) W^{\frac{q}{p^{\prime}}\left(p^{\prime} \beta+1\right)}\left(x_{k+1}\right) \int_{x_{k}}^{x_{k+1}} v(x) W^{q(\alpha-1)}(x) d x
\end{aligned}
$$




$$
\begin{aligned}
= & 2^{3\left(q \beta+\frac{q}{p^{\prime}}\right)}\left(2^{p^{\prime} \beta+1}-1\right)^{\frac{q}{p^{\prime}}} \sum_{k}\left(\int_{x_{k-1}}^{x_{k+1}} f^{p}(s) w(s) d s\right)^{\frac{q}{p}} \\
& \times u^{q}\left(x_{k-1}\right)\left(2^{\left(p^{\prime} \beta+1\right)(k-1)}-2^{\left(p^{\prime} \beta+1\right)(k-2)}\right)^{\frac{q}{p^{\prime}}} \int_{x_{k}}^{x_{k+1}} v(x) W^{q(\alpha-1)}(x) d x \\
\ll & \sum_{k}\left(\int_{x_{k-1}}^{x_{k+1}} f^{p}(s) w(s) d s\right)^{\frac{q}{p}} \\
& \times u^{q}\left(x_{k-1}\right)\left(\int_{x_{k-2}}^{x_{k-1}} W^{p^{\prime} \beta}(s) w(s) d s\right)^{\frac{q}{p^{\prime}}} \int_{x_{k}}^{x_{k+1}} v(x) W^{q(\alpha-1)}(x) d x \\
\ll & \sum_{k}\left(\int_{x_{k-1}}^{x_{k+1}} f^{p}(s) w(s) d s\right)^{\frac{q}{p}} \\
& \times\left(\int_{x_{k-2}}^{x_{k-1}} u^{p^{\prime}}(s) W^{p^{\prime} \beta}(s) w(s) d s\right)^{\frac{q}{p^{\prime}}} \int_{x_{k}}^{x_{k+1}} v(x) W^{q(\alpha-1)}(x) d x \\
& \left(\text { we apply Hölder's inequality with the conjugate exponents } \frac{p}{q}, \frac{p}{p-q}\right) \\
\leq & J_{21}^{\frac{p-q}{p}}\left(\sum_{k} \int_{x_{k-1}}^{x_{k+1}} f^{p}(s) w(s) d s\right)^{\frac{q}{p}} \ll J_{21}^{\frac{p-q}{p}}\|f\|_{p, w}^{q},
\end{aligned}
$$

where

$$
J_{21}=\sum_{k}\left(\int_{x_{k-2}}^{x_{k-1}} u^{p^{\prime}}(s) W^{p^{\prime} \beta}(s) w(s) d s\right)^{\frac{q(p-1)}{p-q}}\left(\int_{x_{k}}^{x_{k+1}} v(x) W^{q(\alpha-1)}(x) d x\right)^{\frac{p}{p-q}} .
$$

To estimate $J_{21}$ we use the relation

$$
\begin{aligned}
& \left(\int_{x_{k-2}}^{x_{k-1}} u^{p^{\prime}}(s) W^{p^{\prime} \beta}(s) w(s) d s\right)^{\frac{q(p-1)}{p-q}} \\
& \quad \ll \int_{x_{k-2}}^{x_{k-1}}\left(\int_{x_{k-2}}^{t} u^{p^{\prime}}(s) W^{p^{\prime} \beta}(s) w(s) d s\right)^{\frac{p(q-1)}{p-q}} u^{p^{\prime}}(t) W^{p^{\prime} \beta}(t) w(t) d t .
\end{aligned}
$$

Then

$$
\begin{aligned}
J_{21} \ll & \sum_{k} \int_{x_{k-2}}^{x_{k-1}}\left(\int_{x_{k-2}}^{t} u^{p^{\prime}}(s) W^{p^{\prime} \beta}(s) w(s) d s\right)^{\frac{p(q-1)}{p-q}} \\
& \times u^{p^{\prime}}(t) W^{p^{\prime} \beta}(t) w(t) d t\left(\int_{x_{k}}^{x_{k+1}} v(x) W^{q(\alpha-1)}(x) d x\right)^{\frac{p}{p-q}} \\
\leq & \sum_{k} \int_{x_{k-2}}^{x_{k-1}}\left(\int_{a}^{t} u^{p^{\prime}}(s) W^{p^{\prime} \beta}(s) w(s) d s\right)^{\frac{p(q-1)}{p-q}} \\
& \times\left(\int_{t}^{b} v(x) W^{q(\alpha-1)}(x) d x\right)^{\frac{p}{p-q}} u^{p^{\prime}}(t) W^{p^{\prime} \beta}(t) w(t) d t \\
\leq & B_{\alpha, \beta}^{\frac{q p}{p-q}} .
\end{aligned}
$$


By substitution of (3.12) in (3.11) we obtain

$$
J_{2} \ll B_{\alpha, \beta}^{q}\|f\|_{p, w}^{q} .
$$

Now, by combining (3.4), (3.10) and (3.13) we obtain

$$
\left\|T_{\alpha, \beta} f\right\|_{q, v} \ll B_{\alpha, \beta}\|f\|_{p, w} .
$$

Consequently, $\left\|T_{\alpha, \beta}\right\|_{q, v} \ll B_{\alpha, \beta}$. The proof is complete.

\section{Compactness of the operator $T_{\alpha, \beta}$}

The main results in this section read as follows.

Theorem 4.1 Let $0<\alpha<1, \frac{1}{\alpha}<p \leq q<\infty$ and $\beta \geq 0$. Let $u$ be a non-increasing function on I. Then the operator $T_{\alpha, \beta}$ is compact from $L_{p, w}$ to $L_{q, v}$ if and only if $A_{\alpha, \beta}<\infty$ and

$$
\lim _{z \rightarrow a^{+}} A_{\alpha, \beta}(z)=\lim _{z \rightarrow b^{-}} A_{\alpha, \beta}(z)=0,
$$

where

$$
A_{\alpha, \beta}(z)=\left(\int_{a}^{z} u^{p^{\prime}}(s) W^{p^{\prime} \beta}(s) w(s) d s\right)^{\frac{1}{p^{\prime}}}\left(\int_{z}^{b} W^{q(\alpha-1)}(x) v(x) d x\right)^{\frac{1}{q}} .
$$

Theorem 4.2 Let $0<\alpha<1, p>\frac{1}{\alpha}$ and $\beta \geq 0$. Let $u$ be a non-increasing function on I. If $b<\infty$ and $0<q<p<\infty$ or $b=\infty$ and $1<q<p<\infty$, then the operator $T_{\alpha, \beta}$ is compact from $L_{p, w}$ to $L_{q, v}$ if and only if $B_{\alpha, \beta}<\infty$.

Proof of Theorem 4.1 Necessity. Let the operator $T_{\alpha, \beta}$ be compact from $L_{p, w}$ to $L_{q, v}$. Then it is bounded and consequently, by Theorem 3.1, we have $A_{\alpha, \beta}<\infty$. First we need to show that $\lim _{z \rightarrow a^{+}} A_{\alpha, \beta}(z)=0$. Consider the family of functions $\left\{f_{t}\right\}_{t \in I}$, where

$$
f_{t}(x)=\chi_{(a, t)}(x) u^{p^{\prime}-1}(x) W^{\left(p^{\prime}-1\right) \beta}(x)\left(\int_{a}^{t} u^{p^{\prime}}(s) W^{p^{\prime} \beta}(s) w(s) d s\right)^{-\frac{1}{p}}, \quad x \in I .
$$

We note that

$$
\begin{aligned}
\left(\int_{a}^{b}\left|f_{t}(x)\right|^{p} w(x) d x\right)^{\frac{1}{p}} & =\left(\int_{a}^{t}\left|f_{t}(x)\right|^{p} w(x) d x\right)^{\frac{1}{p}} \\
= & \left(\int_{a}^{t} u^{p^{\prime}}(s) W^{p^{\prime} \beta}(s) w(s) d s\right)^{-\frac{1}{p}} \\
& \times\left(\int_{a}^{t} u^{p^{\prime}}(s) W^{p^{\prime} \beta}(s) w(s) d s\right)^{\frac{1}{p}}=1 .
\end{aligned}
$$

Next we show that the family of functions $\left\{f_{t}\right\}_{t \in I}$ defined by (4.1) converges weakly to zero in $L_{p, w}$. Let $g \in L_{p^{\prime}, w^{1-p^{\prime}}}=\left(L_{p, w}\right)^{*}$. Then, by Hölder's inequality and (4.2), we find that

$$
\begin{aligned}
\int_{a}^{b} f_{t}(x) g(x) d x & \leq\left(\int_{a}^{t}\left|f_{t}(x)\right|^{p} w(x) d x\right)^{\frac{1}{p}}\left(\int_{a}^{t}|g(s)|^{p^{\prime}} w^{1-p^{\prime}}(s) d s\right)^{\frac{1}{p^{\prime}}} \\
& =\left(\int_{a}^{t}|g(s)|^{p^{\prime}} w^{1-p^{\prime}}(s) d s\right)^{\frac{1}{p^{\prime}}} .
\end{aligned}
$$


Since $g \in L_{p^{\prime}, w^{1-p^{\prime}}}$, the last integral in (4.3) converges to zero as $t \rightarrow a^{+}$, which means weak convergence of the family of functions $\left\{f_{t}\right\}$ to zero as $t \rightarrow a^{+}$. Therefore, from the compactness of the operator $T_{\alpha, \beta}$ from $L_{p, w}$ to $L_{q, v}$ it follows that

$$
\lim _{t \rightarrow a^{+}}\left\|T_{\alpha, \beta} f_{t}\right\|_{q, v}=0
$$

Moreover,

$$
\begin{aligned}
\left\|T_{\alpha, \beta} f_{t}\right\|_{q, v}^{q}= & \int_{a}^{b} v(x)\left(\int_{a}^{x} \frac{u(s) W^{\beta}(s) f_{t}(s) w(s) d s}{(W(x)-W(s))^{1-\alpha}}\right)^{q} d x \\
\geq & \int_{t}^{b} v(x)\left(\int_{a}^{t} \frac{u(s) W^{\beta}(s) f_{t}(s) w(s) d s}{(W(x)-W(s))^{1-\alpha}}\right)^{q} d x \\
\geq & \int_{t}^{b} \frac{v(x) d x}{W^{q(1-\alpha)}(x)}\left(\int_{a}^{t} u^{p^{\prime}}(s) W^{p^{\prime} \beta}(s) w(s) d s\right)^{-\frac{q}{p}} \\
& \times\left(\int_{a}^{t} u^{p^{\prime}}(s) W^{p^{\prime} \beta}(s) w(s) d s\right)^{q} \\
= & A_{\alpha, \beta}^{q}(t) .
\end{aligned}
$$

From (4.4) and (4.5) it follows that $\lim _{t \rightarrow a^{+}} A_{\alpha, \beta}(t)=0$.

Now, we show that $\lim _{t \rightarrow b^{-}} A_{\alpha, \beta}(t)=0$.

From the compactness of the operator $T_{\alpha, \beta}$ from $L_{p, w}$ to $L_{q, v}$ compactness of the conjugate operator follows:

$$
T_{\alpha, \beta}^{*} g(s)=u(s) W^{p}(s) w(s) \int_{s}^{b} \frac{g(x) d x}{(W(x)-W(s))^{1-\alpha}}
$$

from $L_{q^{\prime}, v^{1-q^{\prime}}}$ to $L_{p^{\prime}, w^{1-p^{\prime}}}$.

For $t \in I$ we introduce the family $\left\{g_{t}\right\}_{t \in I}$ of functions:

$$
g_{t}(x)=\chi_{[t, b)}(x)\left(\int_{t}^{b} W^{q(\alpha-1)}(x) v(x) d x\right)^{-\frac{1}{q^{\prime}}} W^{(q-1)(\alpha-1)}(x) v(x) .
$$

The family $\left\{g_{t}\right\}_{t \in I}$ of functions defined by (4.6) is correctly defined, since due to condition $A_{\alpha, \beta}<\infty$ the involving integrals are finite. We show that for all $t \in I$ the functions $g_{t} \in$ $L_{q^{\prime}, v^{1-q^{\prime}}}$ converge weakly to zero as $t \rightarrow b^{-}$.

Indeed,

$$
\begin{aligned}
\left\|g_{t}\right\|_{q^{\prime}, v^{1-q^{\prime}}} & =\left(\int_{t}^{b}\left|g_{t}(x)\right|^{q^{\prime}} v^{1-q^{\prime}}(x) d x\right)^{\frac{1}{q^{\prime}}} \\
& =\left(\int_{t}^{b} W^{q(\alpha-1)}(x) v(x) d x\right)^{-\frac{1}{q^{\prime}}}\left(\int_{t}^{b}\left|W^{(q-1)(\alpha-1)}(x) v(x)\right|^{q^{\prime}} v^{1-q^{\prime}}(x) d x\right)^{\frac{1}{q^{\prime}}} \\
& =\left(\int_{t}^{b} W^{q(\alpha-1)}(x) v(x) d x\right)^{-\frac{1}{q^{\prime}}}\left(\int_{t}^{b} W^{q(\alpha-1)}(x) v(x) d x\right)^{\frac{1}{q^{\prime}}}=1 .
\end{aligned}
$$


By using (4.7) with $f \in L_{q, v}=\left(L_{q^{\prime}, v^{1-q^{\prime}}}\right)^{*}$ we obtain

$$
\begin{aligned}
\int_{a}^{b} g_{s}(x) f(x) d x & \leq\left(\int_{t}^{b}\left|g_{t}(x)\right|^{q^{\prime}} v^{-\frac{q^{\prime}}{q}}(x) d x\right)^{\frac{1}{q^{\prime}}}\left(\int_{t}^{b}|f(x)|^{q} v(x) d x\right)^{\frac{1}{q}} \\
& \leq\left\|g_{t}\right\|_{q^{\prime}, v^{1-q^{\prime}}}\left(\int_{t}^{b}|f(x)|^{q} v(x) d x\right)^{\frac{1}{q}}=\left(\int_{t}^{b}|f(x)|^{q} v(x) d x\right)^{\frac{1}{q}} .
\end{aligned}
$$

Since $f \in L_{q, v}$, the last integral tends to zero as $t \rightarrow b^{-}$, which gives the weak convergence to zero of $\left\{g_{t}\right\}_{t \in I}$ in $L_{q^{\prime}, v^{1-q^{\prime}}}$ as $t \rightarrow b^{-}$. By compactness of $T_{\alpha, \beta}^{*}: L_{q^{\prime}, v^{1-q^{\prime}}} \rightarrow L_{p^{\prime}, w^{1-p^{\prime}}}$ it follows that

$$
\lim _{s \rightarrow b^{-}}\left\|T_{\alpha, \beta}^{*} g_{t}\right\|_{p^{\prime}, w^{1-p^{\prime}}}=0
$$

Furthermore, we note that

$$
\begin{aligned}
\left\|T_{\alpha, \beta}^{*} g_{t}\right\|_{p^{\prime}, w^{1}-p^{\prime}}= & \left(\int_{a}^{b}\left|u(s) W^{\beta}(s) w(s)\right|^{p^{\prime}}\right. \\
& \left.\times\left(\int_{s}^{b} \frac{g_{t}(x) d x}{(W(x)-W(s))^{1-\alpha}}\right)^{p^{\prime}} w^{1-p^{\prime}}(s) d s\right)^{\frac{1}{p^{\prime}}} \\
\geq & \left(\int_{a}^{t} u^{p^{\prime}}(s) W^{p^{\prime} \beta}(s) w(s)\left(\int_{t}^{b} \frac{g_{t}(x) d x}{(W(x)-W(s))^{1-\alpha}}\right)^{p^{\prime}} d s\right)^{\frac{1}{p^{\prime}}} \\
\geq & \left(\int_{a}^{t} u^{p^{\prime}}(s) W^{p^{\prime} \beta}(s) w(s) d s\right)^{\frac{1}{p^{\prime}}}\left(\int_{t}^{b} W^{q(\alpha-1)}(x) v(x) d x\right)^{-\frac{1}{q^{\prime}}} \\
& \times\left(\int_{t}^{b} \frac{W^{(q-1)(\alpha-1)}(x) v(x) d x}{W^{1-\alpha}(x)}\right)^{q}=A_{\alpha, \beta}(t) .
\end{aligned}
$$

Hence, according to (4.8) we have $\lim _{s \rightarrow b^{-}} A_{\alpha, \beta}(s)=0$. The proof of the necessity is complete.

Sufficiency. For $a<c<d<b$ we define

$$
P_{c} f:=\chi_{(a, c)} f, \quad P_{c d} f:=\chi_{(c, d)} f, \quad Q_{d} f:=\chi_{(d, b)} f .
$$

Then

$$
f=P_{c} f+P_{c d} f+Q_{d} f
$$

and since $P_{c} T_{\alpha, \beta} P_{c d} \equiv 0, P_{c} T_{\alpha, \beta} Q_{d} \equiv 0, P_{c d} T_{\alpha, \beta} Q_{d} \equiv 0$, we have

$$
T_{\alpha, \beta} f=P_{c d} T_{\alpha, \beta} P_{c d} f+P_{c} T_{\alpha, \beta} P_{c} f+P_{c d} T_{\alpha, \beta} P_{c} f+Q_{d} T_{\alpha, \beta} f
$$

We show that the operator $P_{c d} T_{\alpha, \beta} P_{c d}$ is compact from $L_{p, w}$ to $L_{q, v}$. Since $P_{c d} T_{\alpha, \beta} P_{c d} f(x)=$ 0 for $x \in I \backslash(c, d)$, it is enough to show that the operator $P_{c d} T_{\alpha, \beta} P_{c d}$ is compact from $L_{p, w}(c, d)$ to $L_{q, v}(c, d)$. This, in turn, is equivalent to compactness of the operator

$$
T f(x)=\int_{c}^{d} K(x, s) f(s) d s
$$


from $L_{p}(c, d)$ to $L_{q}(c, d)$ with the kernel

$$
K(x, s)=\frac{u(s) W^{\beta}(s) v^{\frac{1}{q}}(x) \chi_{(c, d)}(x-s) w^{\frac{1}{p^{\prime}}}(s)}{(W(x)-W(s))^{1-\alpha}} .
$$

Let $\left\{x_{k}\right\}_{k \in \mathbb{Z}}$ be the sequence of points defined in the proof of Theorem 3.1. There are points $x_{i}, x_{n+1}, x_{i}<x_{n+1}$ such that $x_{i} \leq c<x_{i+1}, x_{n}<d \leq x_{n+1}$. We assume that the numbers $c, d$ are chosen so that $x_{i+1}<x_{n}$. Similarly to obtaining estimates of $J_{1}$ and $J_{2}$ in Theorem 3.1, we have

$$
\begin{aligned}
& \int_{c}^{d}\left(\int_{c}^{d}|K(x, s)|^{p^{\prime}} d s\right)^{\frac{q}{p^{\prime}}} d x \\
& \quad=\int_{c}^{d} v(x)\left(\int_{c}^{x} \frac{u^{p^{\prime}}(s) W^{p^{\prime} \beta}(s) w(s) d s}{(W(x)-W(s))^{p^{\prime}(1-\alpha)}}\right)^{\frac{q}{p^{\prime}}} d x \\
& \leq \sum_{k=i}^{n} \int_{x_{k}}^{x_{k+1}} v(x)\left[\left(\int_{a}^{x_{k-1}}+\int_{x_{k-1}}^{x}\right) \frac{u^{p^{\prime}}(s) W^{p^{\prime} \beta}(s) w(s) d s}{(W(x)-W(s))^{p^{\prime}(1-\alpha)}}\right]^{\frac{q}{p^{\prime}}} d x \\
& \leq \mu(n-i+1) A_{\alpha, \beta}^{q}<\infty,
\end{aligned}
$$

where the constant $\mu$ does not depend on $i, n$.

Therefore, on the basis of the Kantarovich condition ([16], p.420), the operator $T$ is compact from $L_{p}(c, d)$ to $L_{q}(c, d)$, which is equivalent to compactness of the operator $P_{c d} T_{\alpha, \beta} P_{c d}$ from $L_{p, w}$ to $L_{q, v}$.

From (4.9) it follows that

$$
\left\|T_{\alpha, \beta}-P_{c d} T_{\alpha, \beta} P_{c d}\right\| \leq\left\|P_{c} T_{\alpha, \beta} P_{c}\right\|+\left\|P_{c d} T_{\alpha, \beta} P_{c}\right\|+\left\|Q_{d} T_{\alpha, \beta}\right\| .
$$

We will show that the right-hand side of (4.10) tends to zero at $c \rightarrow a$ and $d \rightarrow b$. Then the operator $T_{\alpha, \beta}$ as the uniform limit of compact operators is compact from $L_{p, w}$ to $L_{q, v}$. By using Theorem 3.1 we find that

$$
\begin{aligned}
\left\|P_{c} T_{\alpha, \beta} P_{c} f\right\|_{q, v}= & \left(\int_{a}^{c} v(x)\left|\int_{a}^{x} \frac{u(s) W^{\beta}(s) f(s) w(s) d s}{(W(x)-W(s))^{1-\alpha}}\right|^{q} d x\right)^{\frac{1}{q}} \\
\ll & \sup _{a<z<c}\left(\int_{a}^{z} u^{p^{\prime}}(s) W^{p^{\prime} \beta}(s) w(s) d s\right)^{\frac{1}{p^{\prime}}} \\
& \times\left(\int_{z}^{c} v(x) W^{q(\alpha-1)}(x) d x\right)^{\frac{1}{q}}\|f\|_{p, w} \\
\leq & \sup _{a<z<c} A_{\alpha, \beta}(z)\|f\|_{p, w} .
\end{aligned}
$$

Consequently, $\left\|P_{c} T_{\alpha, \beta} P_{c}\right\| \ll \sup _{a<z<c} A_{\alpha, \beta}(z)$. Hence,

$$
\lim _{c \rightarrow a^{+}}\left\|P_{c} T_{\alpha, \beta} P_{c}\right\| \ll \lim _{c \rightarrow a^{+}} \sup _{a<z<c} A_{\alpha, \beta}(z)=\lim _{z \rightarrow a^{+}} A_{\alpha, \beta}(z)=0 .
$$

To estimate $\left\|P_{c d} T_{\alpha, \beta} P_{c}\right\|$ we assume that $v_{\varepsilon}(x)=v(x)$ for $x \in(c, d]$ and $v_{\varepsilon}(x)=\varepsilon^{q} v(x)$ for $x \in(a, c], u_{\varepsilon}(s)=u(s)$ for $s \in(a, c]$ and $u_{\varepsilon}(s)=\varepsilon u(s)$ for $s \in(c, d]$, where $1>\varepsilon>0$. Obviously, 
the function $u_{\varepsilon}$ is non-increasing on $I$. Then, according to Theorem 3.1, we obtain

$$
\begin{aligned}
\left\|P_{c d} T_{\alpha, \beta} P_{c}\right\|_{q, v} & =\left(\int_{c}^{d} v(x)\left|\int_{a}^{c} \frac{u(s) W^{\beta}(s) f(s) w(s) d s}{(W(x)-W(s))^{1-\alpha}}\right|^{q} d x\right)^{\frac{1}{q}} \\
& \leq\left(\int_{a}^{d} v_{\varepsilon}(x)\left|\int_{a}^{x} \frac{u_{\varepsilon}(s) W^{\beta}(s) f(s) w(s) d s}{(W(x)-W(s))^{1-\alpha}}\right|^{q} d x\right)^{\frac{1}{q}} \\
& \ll A_{\alpha, \beta}^{\varepsilon}\|f\|_{p, w},
\end{aligned}
$$

where

$$
A_{\alpha, \beta}^{\varepsilon}=\sup _{a<z<d}\left(\int_{z}^{d} W^{q(\alpha-1)}(x) v_{\varepsilon}(x) d x\right)^{\frac{1}{q}}\left(\int_{a}^{z} u_{\varepsilon}^{p^{\prime}}(s) W^{p^{\prime} \beta}(s) w(s) d s\right)^{\frac{1}{p^{\prime}}} .
$$

We estimate the expression $A_{\alpha, \beta}^{\varepsilon}$ from the above as follows:

$$
\begin{aligned}
A_{\alpha, \beta}^{\varepsilon} \leq & \sup _{a<z<c}\left(\int_{c}^{d} W^{q(\alpha-1)}(x) v(x) d x+\varepsilon^{q} \int_{z}^{c} W^{q(\alpha-1)}(x) v(x) d x\right)^{\frac{1}{q}} \\
& \times\left(\int_{a}^{z} u^{p^{\prime}}(s) W^{p^{\prime} \beta}(s) w(s) d s\right)^{\frac{1}{p^{\prime}}} \\
& +\sup _{c<z<d}\left(\int_{z}^{d} W^{q(\alpha-1)}(x) v(x) d x\right)^{\frac{1}{q}} \\
& \times\left(\int_{a}^{c} u^{p^{\prime}}(s) W^{p^{\prime} \beta}(s) w(s) d s+\varepsilon^{p^{\prime}} \int_{c}^{z} u^{p^{\prime}}(s) W^{p^{\prime} \beta}(s) w(s) d s\right)^{\frac{1}{p^{\prime}}} \\
\leq & \sup _{a<z<c}\left(\int_{c}^{d} W^{q(\alpha-1)}(x) v(x) d x\right)\left(\int_{a}^{z} u^{p^{\prime}}(s) W^{p^{\prime} \beta}(s) w(s) d s\right)+\varepsilon A_{\alpha, \beta} \\
& +\sup _{c<z<d}\left(\int_{z}^{d} W^{q(\alpha-1)}(x) v(x) d x\right)^{\frac{1}{q}}\left(\int_{a}^{c} u^{p^{\prime}}(s) W^{p^{\prime} \beta}(s) w(s) d s\right)^{\frac{1}{p^{\prime}}}+\varepsilon A_{\alpha, \beta} \\
\leq & 2\left(A_{\alpha, \beta}(c)+\varepsilon A_{\alpha, \beta}\right) .
\end{aligned}
$$

Since the left side of (4.12) does not depend on $\varepsilon>0$, substituting (4.13) in (4.12) and letting $\varepsilon \rightarrow 0$, we get

$$
\left\|P_{c d} T_{\alpha, \beta} P_{c} f\right\| \ll A_{\alpha, \beta}(c)\|f\|_{p, w} .
$$

Therefore $\left\|P_{c d} T_{\alpha, \beta} P_{c}\right\| \ll A_{\alpha, \beta}(c)$ and we conclude that

$$
\lim _{c \rightarrow a^{+}}\left\|P_{c d} T_{\alpha, \beta} P_{c}\right\| \ll \lim _{c \rightarrow a^{+}} A_{\alpha, \beta}(c)=0 .
$$

Next, arguing as above we find that

$$
\begin{aligned}
\left\|Q_{d} T_{\alpha, \beta} f\right\|_{q, v} & =\left(\int_{d}^{b} v(x)\left|\int_{a}^{x} \frac{u(s) W^{\beta}(s) f(s) w(s) d s}{(W(x)-W(s))^{1-\alpha}}\right|^{q} d x\right)^{\frac{1}{q}} \\
& \ll \sup _{d<z<b} A_{\alpha, \beta}(z)\|f\|_{p, w} .
\end{aligned}
$$


Consequently,

$$
\lim _{d \rightarrow b^{-}}\left\|Q_{d} T_{\alpha, \beta}\right\| \leq \lim _{d \rightarrow b^{-}} \sup _{d<z<b} A_{\alpha, \beta}(z)=\lim _{z \rightarrow b^{-}} A_{\alpha, \beta}(z)=0 .
$$

From (4.11), (4.14) and (4.15) it follows that the right-hand side of (4.10) tends to zero as $c \rightarrow a^{+}$and $d \rightarrow b^{-}$. The proof is complete.

Proof of Theorem 4.2 In the case $b<\infty$ and $0<q<p<\infty$ the statement of Theorem 4.2 follows from the Ando theorem and its generalizations [17]. Therefore, we only need to prove Theorem 4.2 in the case $a=0, b=\infty$ and $1<q<p<\infty$.

Necessity. Let the operator $T_{\alpha, \beta}$ be compact from $L_{p, w}$ to $L_{q, v}$. Then the operator is bounded. Hence, by Theorem 3.2, $B_{\alpha, \beta}<\infty$.

Sufficiency. Let $B_{\alpha, \beta}<\infty$. Here $T_{\alpha, \beta} f=P_{d} T_{\alpha, \beta} P_{d} f+Q_{d} T_{\alpha, \beta} f$. Therefore

$$
\left\|T_{\alpha, \beta}-P_{d} T_{\alpha, \beta} P_{d}\right\| \leq\left\|Q_{d} T_{\alpha, \beta}\right\| .
$$

Since $d<\infty$, the operator $P_{d} T_{\alpha, \beta} P_{d}$ is compact from $L_{p, w}(0, d)$ to $L_{q, v}(0, d)$, which is equivalent to its compactness from $L_{p, w}$ to $L_{q, v}$. We show that the right-hand side of (4.16) tends to zero as $d \rightarrow \infty$. Then the operator $T_{\alpha, \beta}$ is compact from $L_{p, w}$ to $L_{q, v}$ as the uniform limit of compact operators.

Let $1>\varepsilon>0$. To estimate $\left\|Q_{d} T_{\alpha, \beta} f\right\|$ we suppose that $v_{\varepsilon}(x)=v(x)$ for $x \in[d, \infty)$ and $v_{\varepsilon}(x)=\varepsilon^{q} v(x)$ for $x \in(0, d)$. Using the relations $B_{\alpha, \beta} \approx \widetilde{B}_{\alpha, \beta}$ (see Remark 2.3), in view of Theorem 3.2, we have

$$
\begin{aligned}
\left\|Q_{d} T_{\alpha, \beta} f\right\| & \leq\left(\int_{a}^{\infty} v_{\varepsilon}(x)\left|\int_{a}^{x} \frac{u(s) W^{\beta}(s) f(s) w(s) d s}{(W(x)-W(s))^{1-\alpha}}\right|^{q} d x\right)^{\frac{1}{q}} \\
& \ll \widetilde{B}_{\alpha, \beta}^{\varepsilon}\|f\|_{p, w}
\end{aligned}
$$

or

$$
\left\|Q_{d} T_{\alpha, \beta}\right\| \ll \widetilde{B}_{\alpha, \beta}^{\varepsilon}
$$

where

$$
\begin{aligned}
\widetilde{B}_{\alpha, \beta}^{\varepsilon}= & \left(\int_{a}^{\infty}\left(\int_{z}^{\infty} W^{q(\alpha-1)}(x) v_{\varepsilon}(x) d x\right)^{\frac{q}{p-q}}\right. \\
& \left.\times\left(\int_{a}^{z} u^{p^{\prime}}(s) W^{p^{\prime} \beta}(s) w(s) d s\right)^{\frac{q(p-1)}{p-q}} W^{q(\alpha-1)}(z) v_{\varepsilon}(z) d z\right)^{\frac{p-q}{p q}} .
\end{aligned}
$$

Passing to the limit $\varepsilon \rightarrow 0^{+}$, from (4.17) it follows that

$$
\begin{aligned}
\left\|Q_{d} T_{\alpha, \beta}\right\| \ll & \left(\int_{d}^{\infty}\left(\int_{z}^{\infty} W^{q(\alpha-1)}(x) v(x) d x\right)^{\frac{q}{p-q}}\right. \\
& \left.\times\left(\int_{a}^{z} u^{p^{\prime}}(s) W^{p^{\prime} \beta}(s) w(s) d s\right)^{\frac{q(p-1)}{p-q}} W^{q(\alpha-1)}(z) v(z) d z\right)^{\frac{p-q}{p q}} .
\end{aligned}
$$


Hence,

$$
\lim _{d \rightarrow \infty}\left\|Q_{d} T_{\alpha, \beta}\right\|=0
$$

Obviously, (4.18) implies that the right-hand side of (4.16) tends to zero as $d \rightarrow \infty$. The proof is complete.

\section{Some dual results}

Here we consider the dual operator $K_{\alpha, \beta}^{*}$ defined by

$$
K_{\alpha, \beta}^{*} g(s)=\int_{s}^{b} \frac{u(s) W^{\beta}(s) g(x) v(x) d x}{(W(x)-W(s))^{1-\alpha}}
$$

and its mapping properties from $L_{p, v}$ to $L_{q, w}$.

We define

$$
\begin{aligned}
& A_{\alpha, \beta}^{*}(z):=\left(\int_{a}^{z} u^{q}(s) W^{q \beta}(s) w(s) d s\right)^{\frac{1}{q}}\left(\int_{z}^{b} W^{p^{\prime}(\alpha-1)}(x) v(x) d x\right)^{\frac{1}{p^{\prime}}}, \\
& A_{\alpha, \beta}^{*}=\sup _{z \in I} A_{\alpha, \beta}^{*}(z) .
\end{aligned}
$$

Our first main result here reads as follows.

Theorem 5.1 Let $0<\alpha<1,1<p \leq q<\frac{1}{1-\alpha}$ and $\beta \geq 0$. Let $u$ be a non-increasing function on I. Then the operator $K_{\alpha, \beta}^{*}$ defined by (5.1)

(i) is bounded from $L_{p, v}$ to $L_{q, w}$ if and only if $A_{\alpha, \beta}^{*}<\infty$ and moreover, $\left\|K_{\alpha, \beta}^{*}\right\| \approx A_{\alpha, \beta}^{*}$;

(ii) is compact from $L_{p, v}$ to $L_{q, w}$ if and only if $A_{\alpha, \beta}^{*}<\infty$ and

$$
\lim _{z \rightarrow a^{+}} A_{\alpha, \beta}^{*}(z)=\lim _{z \rightarrow b^{-}} A_{\alpha, \beta}^{*}(z)=0 .
$$

Proof The operator $K_{\alpha, \beta}^{*}$ acting from $L_{p, v}$ to $L_{q, w}$ is conjugate to the operator

$$
K_{\alpha, \beta} f(x)=v(x) \int_{a}^{x} \frac{u(s) W^{\beta}(s) f(s) d s}{(W(x)-W(s))^{1-\alpha}}
$$

acting from $L_{q^{\prime}, w^{1-q^{\prime}}}$ to $L_{p^{\prime}, v^{1-p^{\prime}}}$, which is equivalent to the action of the operator $T_{\alpha, \beta}$ from $L_{q^{\prime}, w}$ to $L_{p^{\prime}, v}$. Consequently, the operator $K_{\alpha, \beta}^{*}$ is bounded and compact from $L_{p, v}$ to $L_{q, w}$ if and only if the operator $T_{\alpha, \beta}$ is, respectively, bounded and compact from $L_{q^{\prime}, w}$ to $L_{p^{\prime}, v}$. Moreover, $\left\|K_{\alpha, \beta}^{*}\right\|=\left\|T_{\alpha, \beta}\right\|$. Since, by the conditions of Theorem 5.1 we have $\frac{1}{\alpha}<q^{\prime} \leq p^{\prime}<\infty$, the statements (i) and (ii) in Theorem 5.1 follow directly from Theorem 3.1 and Theorem 4.1. The proof is complete.

Similarly, in view of Theorem 3.2 we have the following.

Theorem 5.2 Let $0<\alpha<1,1<q<\min \left\{p, \frac{1}{\alpha-1}\right\}, p>1$ and $\beta \geq 0$. Let $u$ be a non-increasing function on $I$. Then the operator $K_{\alpha, \beta}^{*}$ defined by (5.1) is bounded and compact from $L_{p, v}$ to 
$L_{q, w}$ if and only if $B_{\alpha, \beta}^{*}<\infty$, where

$$
\begin{aligned}
B_{\alpha, \beta}^{*}= & \left(\int_{a}^{b}\left(\int_{z}^{b} W^{p^{\prime}(\alpha-1)}(x) v(x) d x\right)^{\frac{q(p-1)}{p-q}}\left(\int_{a}^{z} u^{q}(s) W^{q \beta}(s) w(s) d s\right)^{\frac{q}{p-q}}\right. \\
& \left.\times u^{q}(s) W^{q \beta}(s) w(s) d s\right)^{\frac{p-q}{p q}} .
\end{aligned}
$$

Theorems 5.1 and 5.2 imply especially the following new information in the theory of Hardy type inequalities.

Theorem 5.3 Let $0<\alpha<1, \beta \geq 0$ and $u$ be a non-increasing function on I. Then

$$
\left(\int_{a}^{b}\left(K_{\alpha, \beta}^{*} f(x)\right)^{q} w(x) d x\right)^{\frac{1}{q}} \leq C\left(\int_{a}^{b}(f(x))^{p} v(x) d x\right)^{\frac{1}{p}}
$$

holds if and only if

(a) $A_{\alpha, \beta}^{*}<\infty$ for the case $1<p \leq q \leq \frac{1}{1-\alpha}$,

(b) $B_{\alpha, \beta}^{*}<\infty$ for the case $1<q<\min \left(p, \frac{1}{\alpha-1}\right)$, $p>1$.

Moreover, for the best constant $C$ in (5.2) it yields $C \approx A_{\alpha, \beta}^{*}$ in case (a) and $C \approx B_{\alpha, \beta}^{*}$ in case (b).

Theorem 5.3 supplements the results of [18].

\section{Applications}

By applying our results in special cases we obtain both new and well-known results. Here we just consider the Riemann-Liouville, Erdelyi-Kober, and Hadamard operators mentioned in our introduction. We use the weight functions $\rho$ and $\omega$ and consider these operators on the forms $\widetilde{I}_{\alpha}, \widetilde{E}_{\alpha, \gamma}$ and $\widetilde{\mathcal{H}}_{\alpha}$ defined by

$$
\begin{aligned}
& \widetilde{I}_{\alpha} f(x):=\rho(x)\left[I_{\alpha}(f \omega)\right](x), \\
& \widetilde{E}_{\alpha, \gamma} f(x):=\rho(x)\left[E_{\alpha, \gamma}(f \omega)\right](x), \\
& \widetilde{\mathcal{H}}_{\alpha} f(x):=\rho(x)\left[\mathcal{H}_{\alpha}(f \omega)\right](x),
\end{aligned}
$$

where $\rho$ and $\omega$ are almost everywhere positive functions locally summable on $I$ with degrees $q$ and $p^{\prime}$, respectively.

The action of the operator $T_{\alpha, \beta}$ from $L_{p, v}$ to $L_{q, w}$ is equivalent to the action of the operator

$$
\widetilde{T}_{\alpha, \beta} f(x)=v^{\frac{1}{q}}(x) \int_{a}^{x} \frac{u(s) W^{\beta}(s) w^{\frac{1}{p^{\prime}}}(s) f(s) d s}{(W(x)-W(s))^{1-\alpha}}
$$

from $L_{p}$ to $L_{q}$. Therefore, in the case $W(x)=x$ we have $\rho(x)=v^{\frac{1}{q}}(x), \omega(x)=u(x) x^{\beta}$ and

$$
\widetilde{I}_{\alpha} f(x)=\rho(x) \int_{a}^{x} \frac{\omega(s) f(s) d s}{(x-s)^{1-\alpha}} .
$$


If $W(x)=x^{\sigma}, \sigma>0$, then $u(s) W^{\beta}(s) w^{\frac{1}{p^{\prime}}}(s)=u(s) s^{\sigma \beta-\frac{\sigma-1}{p^{\prime}}}=u(s) s^{\sigma \gamma+\sigma-1}$, where $\gamma=\beta-\frac{\sigma-1}{\sigma p}$. Consequently, $\rho(x)=v^{\frac{1}{q}}(x), \omega(s)=u(s)$ and

$$
\widetilde{E}_{\alpha, \gamma} f(x)=\rho(x) \int_{a}^{x} \frac{\omega(s) s^{\sigma \gamma+\sigma-1} f(s) d s}{\left(x^{\sigma}-s^{\sigma}\right)^{1-\alpha}} .
$$

Now, we assume that $a>0$ and $W(x)=\ln \frac{x}{a}$. Then $u(s) W^{\beta}(s) w^{\frac{1}{p^{\prime}}}(s)=u(s)\left(\ln \frac{s}{a}\right)^{\beta}\left(\frac{a}{s}\right)^{\frac{1}{p^{\prime}}}=$ $a^{\frac{1}{p^{\prime}}} u(s) s^{\frac{1}{p}}\left(\ln \frac{s}{a}\right)^{\beta} \frac{1}{s}$. In this case $\rho(x)=v^{\frac{1}{q}}(x), \omega(s)=u(s) s^{\frac{1}{p}}\left(\ln \frac{s}{a}\right)^{\beta}$ and

$$
\widetilde{\mathcal{H}}_{\alpha} f(x)=\rho(x) \int_{a}^{x} \frac{\omega(s) f(s) d s}{s\left(\ln \frac{x}{s}\right)^{1-\alpha}} .
$$

Below we present statements for boundedness and compactness of the operators $\widetilde{I}_{\alpha}$, $\widetilde{E}_{\alpha, \gamma}$ and $\widetilde{\mathcal{H}}_{\alpha}$ from $L_{p}$ to $L_{q}$. These statements are consequences of Theorems 3.1, 3.2, 4.1, and 4.2 .

We define

$$
\begin{aligned}
& A_{\alpha}^{1}(z):=\left(\int_{z}^{b}\left(\rho(x) x^{\alpha-1}\right)^{q} d x\right)^{\frac{1}{q}}\left(\int_{a}^{z} \omega^{p^{\prime}}(s) d s\right)^{\frac{1}{p^{\prime}}}, \quad A_{\alpha}^{1}:=\sup _{z \in I} A_{\alpha}^{1}(z) \\
& B_{\alpha}^{1}:=\left(\int_{a}^{b}\left(\int_{z}^{b}\left|\rho(x) x^{\alpha-1}\right|^{q} d x\right)^{\frac{p}{p-q}}\left(\int_{a}^{z} \omega^{p^{\prime}}(s) d s\right)^{\frac{p(q-1)}{p-q}} \omega^{p^{\prime}}(z) d z\right)^{\frac{p-q}{p q}}
\end{aligned}
$$

Corollary 6.1 Let $0<\alpha<1, \beta \geq 0$ and $\omega(s)=u(s) s^{\beta}$. Let $u$ be a non-increasing function on I. Then:

(i) for $\frac{1}{\alpha}<p \leq q<\infty$ the operator $\widetilde{I}_{\alpha}$ is bounded from $L_{p}$ to $L_{q}$ if and only if $A_{\alpha}^{1}<\infty$ and, moreover, $\left\|\widetilde{I}_{\alpha}\right\| \approx A_{\alpha}^{1}$; it is compact from $L_{p}$ to $L_{q}$ if and only if $A_{\alpha}^{1}<\infty$ and $\lim _{z \rightarrow a^{+}} A_{\alpha}^{1}(z)=\lim _{z \rightarrow b^{-}} A_{\alpha}^{1}(z)=0$;

(ii) for $0<q<p<\infty$ and $p>\frac{1}{\alpha}$ the operator $\widetilde{I}_{\alpha}$ is bounded (compact if $b<\infty$ or $b=\infty$ and $1 \leq q<p<\infty)$ from $L_{p}$ to $L_{q}$ if and only if $B_{\alpha}^{1}<\infty$.

Remark 6.2 Corollary 6.1 generalizes the results of Theorems 1 and 2, 5 and 6 in [5], where the case $\beta=0$ was considered. Even in this case the results of Corollary 6.1 are different (and in a sense simpler to use) than those in [5], because in [5] the statements are given in terms of two expressions while here we only need one condition.

We define

$$
\begin{aligned}
& A_{\alpha, \gamma}^{2}(z):=\left(\int_{z}^{b}\left|\rho(x) x^{\sigma(\alpha-1)}\right|^{q} d x\right)^{\frac{1}{q}}\left(\int_{a}^{z}\left|\omega(s) s^{\sigma \gamma+\sigma-1}\right|^{p^{\prime}} d s\right)^{\frac{1}{p^{\prime}}}, \\
& A_{\alpha, \gamma}^{2}:=\sup _{z \in I} A_{\alpha, \gamma}^{2}(z), \\
& B_{\alpha, \gamma}^{2}:=\left(\int_{a}^{b}\left(\int_{z}^{b}\left|\rho(x) x^{\sigma(\alpha-1)}\right|^{q} d x\right)^{\frac{p}{p-q}}\right. \\
&\left.\times\left(\int_{a}^{z}\left|\omega(s) s^{\sigma \gamma+\sigma-1}\right|^{p^{\prime}} d s\right)^{\frac{p(p-1)}{p-q}}\left|\omega(z) z^{\sigma \gamma+\sigma-1}\right|^{p^{\prime}} d z\right)^{\frac{p-q}{p q}} .
\end{aligned}
$$


Corollary 6.3 Let $0<\alpha<1, \sigma>0, \beta \geq 0$ and $\gamma=\beta-\frac{\sigma-1}{\sigma p}$. Let $\omega$ be a non-increasing function on I. Then:

(i) for $\frac{1}{\alpha}<p \leq q<\infty$ the operator $\widetilde{E}_{\alpha, \gamma}$ is bounded from $L_{p}$ to $L_{q}$ if and only if $A_{\alpha, \gamma}^{2}<\infty$ and, moreover, $\left\|\widetilde{E}_{\alpha, \gamma}\right\| \approx A_{\alpha, \gamma}^{2}$; it is compact from $L_{p}$ to $L_{q}$ if and only if $A_{\alpha, \gamma}^{2}<\infty$ and $\lim _{z \rightarrow a^{+}} A_{\alpha, \gamma}^{2}(z)=\lim _{z \rightarrow b^{-}} A_{\alpha, \gamma}^{2}(z)=0$;

(ii) for $0<q<p<\infty$ and $p>\frac{1}{\alpha}$ the operator $\widetilde{E}_{\alpha, \gamma}$ is bounded (compact if $b<\infty$ or $b=\infty$ and $1 \leq q<p<\infty)$ from $L_{p}$ to $L_{q}$ if and only if $B_{\alpha, \gamma}^{2}<\infty$.

To formulate statements corresponding to the operator $\tilde{\mathcal{H}}_{\alpha}$ we define

$$
\begin{aligned}
& A_{\alpha}^{3}(z):=\left(\int_{z}^{b}\left|\rho(x)\left(\ln \frac{x}{a}\right)^{\alpha-1}\right|^{q} d x\right)^{\frac{1}{q}}\left(\int_{a}^{z} \omega^{p^{\prime}}(s) d s\right)^{\frac{1}{p^{\prime}}}, \quad A_{\alpha}^{3}:=\sup _{z \in I} A_{\alpha}^{3}(z), \\
& B_{\alpha}^{3}:=\left(\int_{a}^{b}\left(\int_{z}^{b}\left|\rho(x)\left(\ln \frac{x}{a}\right)^{\alpha-1}\right|^{q} d x\right)^{\frac{p}{p-q}}\left(\int_{a}^{z} \omega^{p^{\prime}}(s) d s\right)^{\frac{p(q-1)}{p-q}} \omega^{p^{\prime}}(z) d z\right)^{\frac{p-q}{p q}} .
\end{aligned}
$$

Corollary 6.4 Let $a>0,0<\alpha<1, \beta \geq 0$ and $\omega(s)=u(s) s^{\frac{1}{p}}\left(\ln \frac{s}{a}\right)^{\beta}$. Let $u$ be a nonincreasing function on $I$. Then:

(i) for $\frac{1}{\alpha}<p \leq q<\infty$ the operator $\widetilde{\mathcal{H}}_{\alpha}$ is bounded from $L_{p}$ to $L_{q}$ if and only if $A_{\alpha}^{3}<\infty$ and, moreover, $\left\|\widetilde{\mathcal{H}}_{\alpha}\right\| \approx A_{\alpha}^{3}$; it is compact from $L_{p}$ to $L_{q}$ if and only if $A_{\alpha}^{3}<\infty$ and $\lim _{z \rightarrow a^{+}} A_{\alpha}^{3}(z)=\lim _{z \rightarrow b^{-}} A_{\alpha}^{3}(z)=0$;

(ii) for $0<q<p<\infty$ and $p>\frac{1}{\alpha}$ the operator $\widetilde{\mathcal{H}}_{\alpha}$ is bounded (compact if $b<\infty$ or $b=\infty$ and $1 \leq q<p<\infty)$ from $L_{p}$ to $L_{q}$ if and only if $B_{\alpha}^{3}<\infty$.

Finally, we consider the operator $\widetilde{I}_{\alpha}^{*} g(s)=\rho(s)\left[I_{\alpha}^{*}(g \omega)\right](s), s \in I$, acting from $L_{p}$ to $L_{q}$, where $I_{\alpha}^{*}$ is the Weyl operator

$$
I_{\alpha}^{*} g(s)=\int_{s}^{b} \frac{g(x) d x}{(x-s)^{1-\alpha}}
$$

The action of the operator $K_{\alpha, \beta}^{*}$ from $L_{p, v}$ to $L_{q, w}$ is equivalent to the action of the operator

$$
\widetilde{K}_{\alpha, \beta}^{*} g(s)=w^{\frac{1}{q}}(s) u(s) W^{\beta}(s) \int_{s}^{b} \frac{v^{\frac{1}{p^{\prime}}}(x) g(x) d x}{(W(x)-W(s))^{1-\alpha}}
$$

from $L_{p}$ to $L_{q}$. Therefore, when $W(x)=x$ we have

$$
\begin{aligned}
& \rho(s)=u(s) s^{\beta}, \quad \omega(x)=v^{\frac{1}{p^{\prime}}}(x), \\
& \widetilde{I}_{\alpha}^{*} g(s)=\rho(s) \int_{s}^{b} \frac{\omega(x) g(x) d x}{(x-s)^{1-\alpha}} .
\end{aligned}
$$

We define

$$
\begin{aligned}
& A_{\alpha}^{*}(z):=\left(\int_{a}^{z} \rho^{q}(s) d s\right)^{\frac{1}{q}}\left(\int_{z}^{b}\left|\omega(x) x^{\alpha-1}\right|^{p^{\prime}} d x\right)^{\frac{1}{p^{\prime}}}, \quad A_{\alpha}^{*}:=\sup _{z \in I} A_{\alpha}^{*}(z), \\
& B_{\alpha}^{*}:=\left(\int_{a}^{b}\left(\int_{z}^{b}\left|\omega(x) x^{\alpha-1}\right|^{p^{\prime}} d x\right)^{\frac{q(p-1)}{p-q}}\left(\int_{a}^{z} \rho^{q}(s) d s\right)^{\frac{q}{p-q}} \rho^{q}(z) d z\right)^{\frac{p-q}{p q}} .
\end{aligned}
$$


From Theorems 5.1 and 5.2 we have the following result.

Corollary 6.5 Let $0<\alpha<1, \beta \geq 0$ and $\rho(s)=u(s) s^{\beta}$. Let $u$ be a non-increasing function on I. Then:

(i) for $1<p \leq q<\frac{1}{1-\alpha}$ the operator $\widetilde{I}_{\alpha}^{*}$ is bounded from $L_{p}$ to $L_{q}$ if and only if $A_{\alpha}^{*}<\infty$ and, moreover, $\left\|\widetilde{I}_{\alpha}\right\| \approx A_{\alpha}^{*}$; it is compact from $L_{p}$ to $L_{q}$ if and only if $A_{\alpha}^{*}<\infty$ and $\lim _{z \rightarrow a^{+}} A_{\alpha}^{*}(z)=\lim _{z \rightarrow b^{-}} A_{\alpha}^{*}(z)=0$;

(ii) for $1<q<\left\{\min \left(p, \frac{1}{1-\alpha}\right)\right\}<\infty$ and $p>1$ the operator $\widetilde{I}_{\alpha}^{*}$ is bounded (compact) from $L_{p}$ to $L_{q}$ if and only if $B_{\alpha}^{*}<\infty$.

Remark 6.6 From the results in Corollary 6.1-6.4 follow some corresponding Hardy type inequalities, which seem to be new even as they are special cases of our Theorems 3.3 and 5.3.

Competing interests

The authors declare that they have no competing interests.

Authors' contributions

All the authors contributed equally and significantly in writing this paper. All the authors read and approved the final manuscript.

\section{Author details}

'Department of Mechanics and Mathematics, L.N. Gumilyov Eurasian National University, 2 Satpaev St., Astana, 010008, Kazakhstan. ${ }^{2}$ Department of Engineering Sciences and Mathematics, Luleå University of Technology, Luleå, 97187 , Sweden. ${ }^{3}$ UiT, The Artic University of Norway, Troms $\varnothing$, Norway.

\section{Acknowledgements}

We thank both referees for very good remarks, which have helped us to improve the final version of this paper.

Received: 2 September 2016 Accepted: 29 November 2016 Published online: 13 December 2016

\section{References}

1. Samko, SG, Kilbas, AA, Marichev, Ol: Integrals and Derivatives of Fractional Order and Some of Their Applications. Science and Technology, Minsk (1987) (in Russian)

2. Stepanov, VD: Two-weighted estimates of Riemann-Liouville integrals. Proc. USSR Acad. Sci., Izv. AN SSSR Ser. Mat. 54(3), 645-655 (1990) (in Russian)

3. Stepanov, VD: On a weighted inequality of Hardy type for fractional Riemann-Liouville integrals. Sib. Math. J. 31(3), 186-197 (1990) (in Russian)

4. Andersen, KF, Sawyer, ET: Weighted norm inequalities for the Riemann-Liouville and Weyl fractional integral operators. Trans. Am. Math. Soc. 308, 547-558 (1988)

5. Farsani, SM: On the boundedness and compactness of the fractional Riemann-Liouville operators. Sib. Mat. Zh. 54(2), 468-479 (2013) (in Russian); translation in Sib. Math. J. 54(2), 368-378 (2013)

6. Lorente, M: A characterization of two weighted norm inequalities for one-sided operators of fractional type. Can. J. Math. 49, 1010-1033 (1997)

7. Meskhi, A: Solution of some weight problems for the Riemann-Liouville and Weyl operators. Georgian Math. J. 106 727-733 (1989)

8. Prokhorov, DV: On the boundedness and compactness of a class of integral operators. J. Lond. Math. Soc. 61(2), 617-628 (2000)

9. Prokhorov, DV, Stepanov, VD: Weighted estimates for the Riemann-Liouville operators and applications. Proc. Math. Inst. RAS Steklov V.A. 248, 289-312 (2003) (in Russian)

10. Oinarov, R: Two-sided estimates of the norms for certain classes of integral operators. Proc. Math. Inst. RAS Steklov V.A. 204, 240-250 (1993) (in Russian)

11. Abylayeva, AM, Kaskirbaeva, D: Boundedness and compactness of fractional integral operator type Holmgren in weighted Lebesgue spaces. Eurasian Math. J. 2, 75-86 (2007) (in Russian)

12. Oinarov, R, Abylayeva, AM: Criteria for the boundedness of a class of fractional integral operators. Math. J. 4(2(12)) 5-14 (2004) (in Russian)

13. Kufner, A, Maligranda, L, Persson, LE: The Hardy Inequality. About Its History and Some Related Results. Vydavatelsky Servis Publishing House, Pilsen (2007)

14. Kufner, A, Persson, L-E: Weighted Inequalities of Hardy Type. World Scientific, Singapore (2003)

15. Sinnamon, G, Stepanov, VD: The weighted Hardy inequality: new proofs and the case $p=1$. J. Lond. Math. Soc. 54 89-101 (1996)

16. Kantarovich, LV, Akilov, GR: Functional Analysis. Nauka, Moscow (1977) (in Russian)

17. Krasnosel'skii, MA, Zabreiko, PP, Pustilnik, EN, Sobolewski, PE: Integral Operators in Spaces of Summable Functions. Nauka, Moscow (1966) (in Russian)

18. Abylayeva, AM: Boundedness, compactness for a class of fractional integral operators of Weyl type. Eurasian Math. J. 7(1), 9-27 (2016) 